CASE REPORTS

\title{
Ultrasonographic search for hidden hygroscopic dilators
}

\author{
Shiyo Ota, J un Sasahara, Keisuke I shii, Nobuaki Mitsuda \\ Osaka Medical Center and Research Institute for Maternal and Child Health, Osaka, Japan
}

Correspondence: Shiyo Ota. Address: Osaka Medical Center and Research Institute for Maternal and Child Health, Osaka, Japan. Email: shiyo0218@yahoo.co.jp

Received: April 16, 2015

Accepted: May 12, 2015

Online Published: June 16, 2015

DOI : $10.5430 /$ ijdi.v2n2p70

URL: http://dx.doi.org/10.5430/ijdi.v2n2p70

\section{Abstract}

Hygroscopic dilators are considered to offer a safe and effective tool for ripening the uterine cervix, usually for the purposes of cervical ripening. We experienced the case of dilator migration into the uterine cavity. Transvaginal ultrasonography seems to be useful to rapidly confirm the location of the cervical dilator under these situations.

\section{Keywords}

Hygroscopic dilator, Transvaginal ultrasonography, Spontaneous migration

\section{I ntroduction}

Hygroscopic dilators are considered to offer a safe and effective tool for ripening the uterine cervix, usually for the purposes of cervical ripening. Following transvaginal ultrasonographic evaluation of the uterine configuration and manual pelvic examination, hygroscopic dilators are placed in the cervical canal. The hygroscopic dilators subsequently absorb endocervical fluids for several hours and become swollen, resulting in sufficient expansion of the cervical canal and thus facilitating the subsequent procedure ${ }^{[1]}$.

\section{Case report}

The present report describes the case of a 26-year-old woman who underwent dilation of the uterine cervix with three hygroscopic dilators (Dilapan-S®; MEDICEM Technology, Czech Republic), for artificial abortion at 17 weeks of gestation. Fourteen hours after placement of the dilators, the patient experienced discomfort around the vaginal cavity. Vaginoscopy indicated the presence of only 1 dilator in the vagina, and the other two dilators could not be detected. However, transvaginal ultrasonography identified the other two dilators as hypoechogenic objects within the uterine cavity, located adjacent to the fetus (see Figure 1). After conservative management for $14 \mathrm{~h}$, these two dilators spontaneously migrated into the vagina. 
Figure 1. Transvaginal ultrasonography showing two Dilapan-S® dilators, located adjacent to the fetus, as hypoechoic objects in the uterine cavity

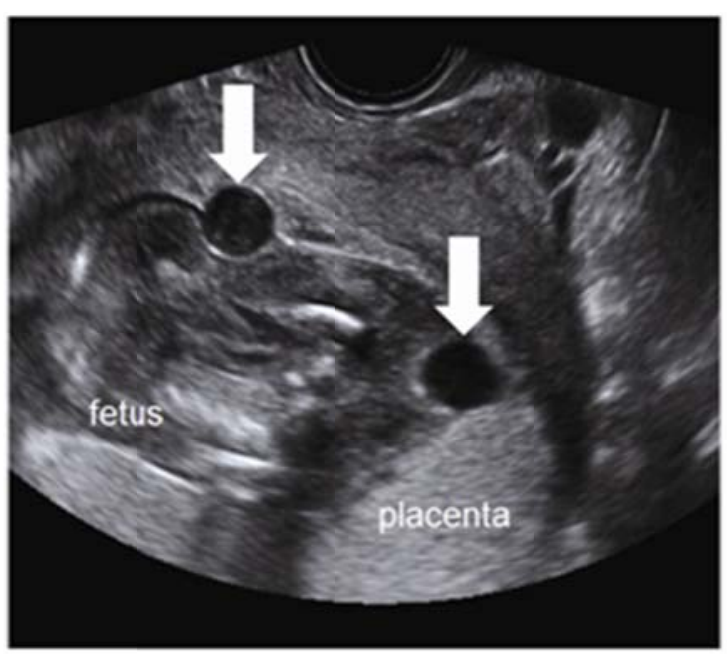

\section{Discussion}

Only a few cases of dilator migration into the uterine cavity have been described previously. Lichtenberg report the case of a patient with a ten-year history of pelvic pain and bleeding resulting from retention of a nearly entire Dilapan-S® dilator ${ }^{[2]}$. This is why early detection for lost hygroscopic dilators is required. Transvaginal ultrasonography seems to be useful to rapidly confirm the location of the cervical dilator under these situations.

In the present case, a portion of each dilator was visible at the external os on initial insertion into the cervical canal. The mechanism underlying migration into the uterine cavity thus remains unclear. Dilapan-S ${ }^{\circledR}$ dilators are composed of hydrophilic polymer, and can therefore be identified ultrasonographically as a hypoechoic object, with similar echogenicity to water.

\section{Conclusion}

In cases where a cervical dilator cannot be located, ultrasonography is likely to prove useful to confirm the location.

\section{Human rights statements and informed consent}

All procedures followed were in accordance with the ethical standards of the responsible committee on human experimentation (institutional and national) and with the Helsinki Declaration of 1975, as revised in 2008 (5). Informed consent was obtained from all patients for being included in the study.

\section{References}

[1] Jozwiak M, Bloemenkamp KW, Kelly AJ, et al. Mechanical methods for induction of labour. The Cochrane Database of Systematic Reviews. 2012 Mar 14; 3: CD001233.

[2] Lichtenberg ES. Complications of osmotic dilators. Obstetrical \& Gynecological Survey. 2004; 59(7): 528-36. 